\title{
Demo Abstract: Real-time Attitude and Motion Tracking for Mobile Device in Moving Vehicle
}

\author{
Chongguang Bi \\ Michigan State University
}

\author{
Guoliang Xing \\ The Chinese University of Hong Kong
}

\begin{abstract}
Recently a class of new in-vehicle technologies based on off-theshelf mobile devices have been developed to improve driver safety and driving experience. For instance, smartwatches are utilized to monitor driving performance and detect possible secondary tasks of drivers such as texting, operating the in-vehicle infotainment, or eating. However, a key challenge for these systems is to track the real-time attitude of mobile devices in a driving vehicle. This demo presents a novel system called Real-time Attitude and Motion Tracking (RAMT) that can enable a mobile device to accurately learn the coordinate system of the moving vehicle, and hence track the attitude and the motion of the device in real time.
\end{abstract}

\section{CCS CONCEPTS}

- Human-centered computing $\rightarrow$ Mobile devices; Smartphones; Empirical studies in ubiquitous and mobile computing;

\section{KEYWORDS}

Wearables, Internet-of-Things

\section{ACM Reference format:}

Chongguang Bi and Guoliang Xing. 2018. Demo Abstract: Real-time Attitude and Motion Tracking for Mobile Device in Moving Vehicle. In Proceedings of SenSys '18: Conference on Embedded Networked Sensor Systems, Shenzhen, China, November 4-7, 2018 (SenSys '18), 2 pages.

https://doi.org/10.1145/3274783.3275181

\section{INTRODUCTION}

Recent years have witnessed the emergence of a class of new invehicle technologies for improving driver safety and driving experience. A number of proprietary in-vehicle technologies have been deployed to monitor the driver's performance. Several systems utilize smartwatches to monitor driving performance and detect possible second tasks of drivers such as texting, operating the invehicle infotainment, or eating [4] [1]. However, these systems often have poor performance due to the significant dynamics of moving vehicles. In particular, the motion of the smartwatch worn by the driver is induced by the movement of both driver's hand and the vehicle. As a result, it is challenging to use the motion sensors of the smartwatch to track driver hand motion or recognize the gesture accurately in real time. A possible solution to address these

Permission to make digital or hard copies of all or part of this work for personal or classroom use is granted without fee provided that copies are not made or distributed for profit or commercial advantage and that copies bear this notice and the full citation on the first page. Copyrights for components of this work owned by others than ACM must be honored. Abstracting with credit is permitted. To copy otherwise, or republish, to post on servers or to redistribute to lists, requires prior specific permission and/or a fee. Request permissions from permissions@acm.org.

SenSys '18, November 4-7, 2018, Shenzhen, China

(C) 2018 Association for Computing Machinery.

ACM ISBN 978-1-4503-5952-8/18/11 . \$ \$15.00

https://doi.org/10.1145/3274783.3275181 issues is leveraging GPS and compass [2] [5] to compute the vehicle's moving direction and the device's attitude relative to the earth, which can then be combined to derive the device's attitude in the vehicle. However, the GPS signal is not always available or reliable. Moreover, the low sampling rate of GPS (around $1 \mathrm{~Hz}$ ) makes it impractical to track hand gestures in real time. The compass often fails to achieve accurate measurement because of the magnetic interference from the vehicle or other electronic devices [3].

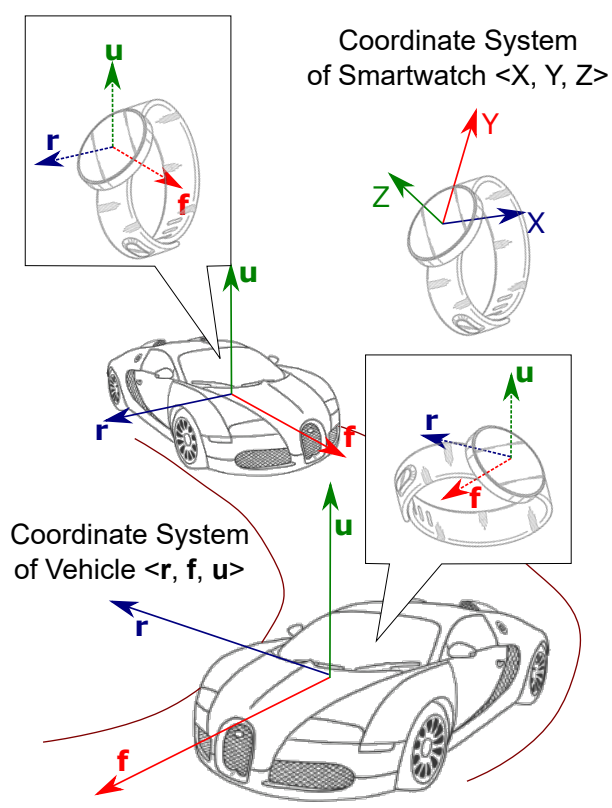

Figure 1: The coordinate system of a mobile device is represented as $\langle X, Y, Z\rangle$. Our goal is to find and maintain a set of unit vectors $<r, f, u>$ under the coordinate systems of the mobile device, and those vectors always point to the forward, right, and up directions of the vehicle.

In this work, we propose a novel system called Real-time Attitude and Motion Tracking (RAMT) that can enable a mobile device to accurately learn the coordinate system of the moving vehicle, and hence track the attitude and the motion of the device in real time. As shown in Fig. 1, our goal is to let the devices learn the vehicle's forward, right, and up directions, and represent these directions under the devices' own coordinate systems as unit vectors $\boldsymbol{r}, \boldsymbol{f}$, and $\boldsymbol{u}$. By sensing the vehicle's movement and calculating its impact on the motion sensors, the mobile device can extract its own motion and hence track the hand movement and recognize gestures. However, a key challenge lies in the fact that computing the accurate alignment between the coordinate systems of the mobile device and the vehicle poses prohibitively high overhead. To 


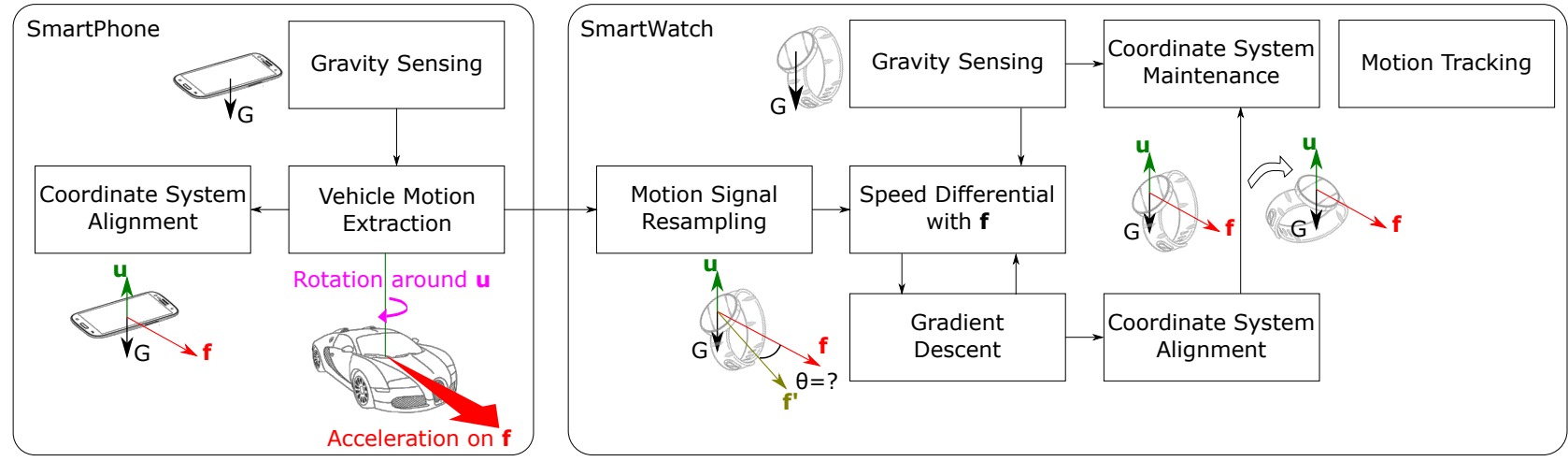

Figure 2: The pipeline of RAMT.

address this challenge, we propose a light-weight algorithm that allows the smartwatch to efficiently search for the alignment between the coordinate systems of the vehicle and itself. The alignment of coordinate systems then allows a smartwatch to extract a user's hand motion. Our system is light-weight and can be run in real time on mobile devices without the communication with the cloud.

\section{SYSTEM OVERVIEW}

RAMT targets to align the coordinate system of each mobile device to that of the vehicle. To learn the attitude and track the motion of the mobile devices, e.g. a smartphone and a smartwatch, we process and analyze the fused motion signals from both devices. The smartphone, which is not being used while driving, is able to pick up the vehicle's accelerating, braking and turning. On the other side, the motion data collected by the smartwatch contains not only the vehicle's motion but also the driver's hand motion. Our key idea is that the smartwatch receives the information about the vehicle's motion from the smartphone via the Bluetooth, and uses this information to learn the attitude of itself, then extracts the hand motion from the mixed motion signal.

Fig. 2 illustrates RAMT's pipeline. Each device calculates the up direction of the vehicle individually by sensing the gravity. The smartphone senses the vehicle's movement and calculates the forward direction, then sends these information to the smartwatch via the Bluetooth. Then, the smartwatch calculates the vehicle's forward direction based on the received information and recognizes its attitude in the vehicle. By re-sampling the received vehicle's motion, the smartwatch compares the motion data collected by itself with the vehicle's motion. Then, we use the gradient descent method to minimize the differential of the calculated speed between the smartwatch and the vehicle, which searches for the best alignment between the two coordinate systems. Finally, the forward and the right directions of the vehicle are determined and maintained continuously by the smartwatch along with the vehicle's turning. The motion tracking of the smartwatch is performed simultaneously based on its real-time attitude.

\section{DEMO SETUP}

For the demonstration purpose, we implemented the system in an App on a smartwatch (Moto 360) and a smartphone (Moto G). When they are carried by the user, the user's walking and turning can simulate the vehicle's movement. The real-time tracking result of
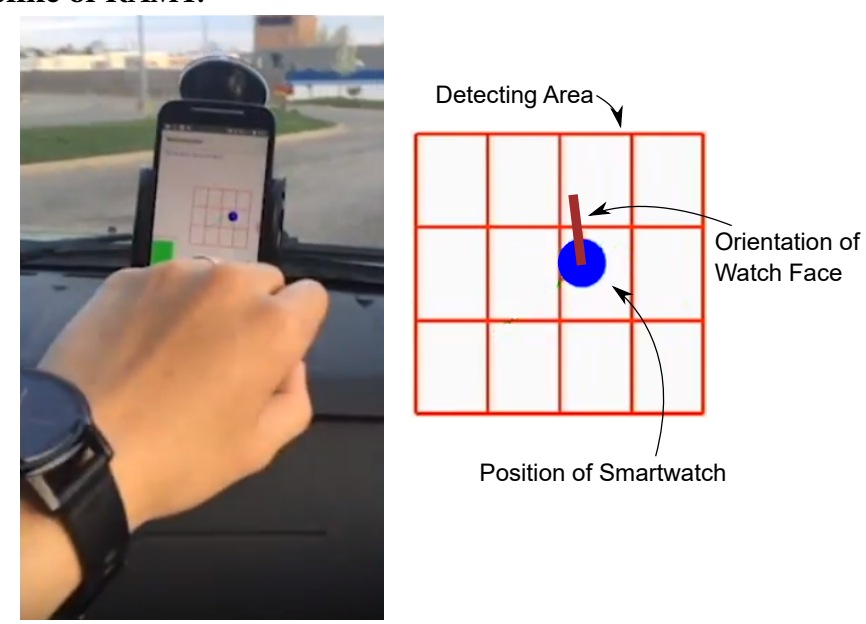

Figure 3: The demonstration of RAMT. The detecting area is the space in front of the user. The real-time tracking result of the smartwatch is displayed as a blue circle on the screen of the smartphone. The orientation of the watch face is represented as a red line segment.

the smartwatch is displayed on the screen of the smartphone as shown in Fig. 3.

\section{ACKNOWLEDGMENTS}

This work was supported in part by U.S. National Science Foundation under grant IIS 1622659.

\section{REFERENCES}

[1] Chongguang Bi, Jun Huang, Guoliang Xing, Landu Jiang, Xue Liu, and Minghua Chen. 2017. SafeWatch: A Wearable Hand Motion Tracking System for Improving Driving Safety. In Internet-of-Things Design and Implementation (IoTDI), 2017 IEEE/ACM Second International Conference on. IEEE, 223-232.

[2] Paul D Groves. 2013. Principles of GNSS, inertial, and multisensor integrated navigation systems. Artech house.

[3] Hua Huang and Shan Lin. 2016. Toothbrushing monitoring using wrist watch. In Proceedings of the 14th ACM Conference on Embedded Network Sensor Systems $C D-R O M$. ACM, 202-215.

[4] Landu Jiang, Xinye Lin, Xue Liu, Chongguang Bi, and Guoliang Xing. 2018. SafeDrive: Detecting Distracted Driving Behaviors Using Wrist-Worn Devices. Proceedings of the ACM on Interactive, Mobile, Wearable and Ubiquitous Technologies 1, 4 (2018), 144

[5] Cagdas Karatas, Luyang Liu, Marco Gruteser, and Richard Howard. 2018. Singlesensor Motion and Orientation Tracking in a Moving Vehicle. In 2018 15th Annual IEEE International Conference on Sensing, Communication, and Networking (SECON). IEEE 